\title{
New flu treatments only a sniff away
}

The choices of treatments and preventions for influenza will be much wider early in the next millennium, and possibly even sooner. Aviron (Mountain View, CA), BioChem Pharma (Laval, Quebec, Canada), Merck (Whitehouse Station, NJ), Chiron (Emeryville, CA), and Cortecs (London), will all have influenza vaccines in the clinic during the 1997-1998 flu season, with some of them given intranasally or orally. Meanwhile, GlaxoWellcome (London) and the team of Gilead Sciences (Foster City, CA) and Hoffmann-La Roche (Basel, Switzerland) are testing neuraminidase inhibitors that block the replication of the influenza virus.

Influenza viruses infect approximately 120 million people in the world's richest countries each year, many more during flu pandemics. Type A influenza causes about $70 \%$ of these cases, type B the rest. Vaccine developers are vying for entry into an estimated $\$ 600$ million worldwide market while the potential market in the US alone for prescription drugs to treat influenza approaches $\$ 900$ million per year.

There is now evidence from phase II clinical trials that neuraminidase inhibitors can diminish the severity of influenza symptoms if taken early in the course of infection. GlaxoWellcomes's zanamivir (GG167) is at least as effective as two currently available antiinfluenza agents, amantadine and rimantadine (New Engl. J. Med. 337:874-880; 1997) compounds that seem only effective against type A influenza strains, cause side effects, and which are already eliciting drug resistant viral strains. Reports from the US Centers for Disease Control and Prevention (Atlanta, GA) suggest that type B viruses may predominate this winter. Zanamivir is active against a range of influenza $\mathrm{A}$ and $\mathrm{B}$ viruses in vitro.

In two parallel trials in North America and Europe, GlaxoWellcome demonstrated that zanamivir, when given as an inhaled powder within 48 hours of the appearance of influenza symptoms, shortened the median time to alleviation of major symptoms by one day, and by three days among the subgroup of patients who began drug treatment within 30 hours, or who had fever at entry into the study. Treated patients were infec-

Vicki Glaser is a freelance writer working in Allentown, PA. tious for only four days, rather than the six days of untreated patients. Phase III trials of zanamivir are now taking place in the southern hemisphere and will begin this flu season in North America and Europe.

Gilead's drug is an oral neuraminidase inhibitor, GS 4104. In a phase II trial, volunteers received the drug 28 hours after intranasal exposure to influenza A. Viral titers in the treatment group were more than 100 times lower than in the placebo group 24 hours after treatment began. Disease symptoms ceased in just over 2 days on average, compared with just under 4 days for the untreated group. The drug could also apparently prevent infection if given up to 26 hours before the virus was administered. Patients given GS 4104 had no detectable nasal virus, whereas $50 \%$ of the placebo group did. In the 1997-1998 influenza season, the Gilead/Roche drug will enter phase III studies on natural influenza infections.

Another neuraminidase inhibitor, from BioCryst Pharmaceuticals (Birmingham, $\mathrm{AL}$ ), is in preclinical studies, $12-18$ months behind the Glaxo and Gilead products.

Clearly, early diagnosis will be important in allowing the neuraminidase inhibitors to be effective. Glaxo is working on an officebased flu diagnostic, as is ZymeTx (Oklahoma City, OK). ZymeTx's ViraZyme, substrates that selectively react with conserved catalytic sites on neuraminidase, emit a color signal to indicate viral infection. The test yieids results within an hour. ZymeTx estimates the market for a "doctor's office" rapid influenza diagnostic at $\$ 200-\$ 250$ million.

Aviron's intranasal vaccine is a live attenuated virus that expresses antigens from the epidemiolgically predicted next wave of virulent virus. It stimulates an antibody response in the nasal mucosa, and was successful in preventing influenza in children, protecting $93 \%$ of $1-6$ year-olds with culture-confirmed influenza. With conventional vaccines, children often require two doses to trigger a protective immune response. The vaccine will now enter phase III clinical trials in healthy working adults and in children who have asthma.

The company also plans to test its vaccine in combination with the classic inactivated influenza injection to boost protection in individuals at high risk of complications from infection. Aviron is likely to apply for a product license from the FDA in mid-1998 and is planning to make the vaccine widely available by the 1999-2000 flu season.

Chiron Biocine's influenza vaccine incorporating the MF59 immunostimulant already has approval in Italy. Chiron will institute a postmarketing trial, and will continue phase III trials in the US.

Producing an influenza vaccine based on virus grown in cell culture, rather than in fertilized chicken eggs, is one facet of the $\mathrm{R} \& \mathrm{D}$ effort at BioChem Pharma. Use of cell culture technology could eliminate problems of inadequate vaccine supply during pandemics, and would make the vaccine safe for people who are allergic to eggs. The company completed a phase I trial in Canada during the last flu season, and will undertake a phase III trial with an injectable cell culturebased vaccine this season. Phase I data demonstrated a signficant boost in immunity in healthy volunteers against both $\mathrm{A}$ and $\mathrm{B}$ strains.

BioChem Pharma's next goal is to package the vaccine for nasal administration, using delivery technology licensed from Biovector Therapeutics (Toulouse, France). "Biovectors" are polysaccharide cores surrounded by a phospholipid layer. In vaccine formulations, the company says, they adhere to mucous membranes, protect the viral antigen, and can enhance its activity. BioChem Pharma will first test nasal administration of its traditional inactivated influenza vaccine, Fluviral S/F, and will then combine nasal delivery with the cell-cultured vaccine. The intranasal form will enter phase I trials this flu season.

"The nasal vaccine can be self-administered," points out Claude Vezeau, president of the BioChem Vaccines division, so that even if the product itself costs more than traditional vaccines, cost savings would accrue from eliminating the need for a visit to the doctor.

Other vaccines are at earlier stages of development. Cortecs recently received a US patent for its Flustat oral influenza vaccine, which is in phase I trials led by the Australian Institute of Mucosal Immunology (Newcastle, Australia). Preliminary results of the escalating dose study have documented local and systemic immune responses. Cortecs attaches killed influenza virus to anucleated red blood cells, which are taken up by immune tissue in the intestine, resulting, Cortecs says, in mucosal immunity to a broad range of viral strains in the respiratory tract. Also in phase I trials is Merck's naked DNA influenza vaccine vaccine, licensed from Vical (San Diego, CA).

Vicki Glaser 